\title{
A Rare Case of Sporadic Inclusion Body Myositis with Atypical Presentation
}

\author{
Manokaran Chinnusamy ${ }^{1}$ Sathiyanarayanan Janakiraman ${ }^{1}$ Ramesh Bala Arivazhagan ${ }^{1}$ \\ 1 Department of General Medicine, Sri Manakula Vinayagar Medical \\ College and Hospital, Madagadipet, Kalitheerthalkuppam, \\ Pondicherry, India \\ J Health Allied Sci ${ }^{\mathrm{NU}}$ 2022;12:333-335. \\ Address for correspondence Manokaran Chinnusamy, MD, \\ Department of General Medicine, 9, Associate Professor Quarters, \\ SMVMCH Campus, Kalitheerthalkuppam, Madagadipet, Pondicherry \\ 605107, India \\ (e-mail: manokaran.smvmch@gmail.com).
}

\begin{abstract} Keywords

- sporadic inclusion body myositis

- endomysial lymphonuclear infiltrates

- rimmed vacuoles

- fat infiltration

- creatine kinase

Sporadic inclusion body myositis (IBM) is the most common acquired inflammatory myopathy that occurs after the age of 50 years. IBM typically involves wrist and finger flexors and quadriceps, but all sporadic IBM may not have the classic presentation of distal arm and proximal leg involvement. Treating physicians must be aware of this atypical presentation to avoid the misdiagnosis of IBM, leading to treatment with immunosuppressive agents. The aim of this study is to increase the awareness among physicians about the atypical presentation of IBM and to emphasize the importance of muscle biopsy in such cases. Here we report a case of 52 years old male diagnosed with sporadic IBM by muscle biopsy presented with atypical presentation.
\end{abstract}

\section{Introduction}

Sporadic inclusion body myositis (IBM) is the most common acquired muscle disease in patients older than 50 years of age. ${ }^{1}$ It is becoming more prevalent because of the increasing age of the population, the emerging development of more inclusive diagnostic criteria, and the advent of a diagnostic autoantibody. Prevalence of sporadic IBM ranges from 5 per million to 71 per million, while this variability may relate to geographic location. ${ }^{2}$

In most cases, the presenting symptoms are related to weakness of the quadriceps femoris and other proximal lower limb muscles. However, some patients have unusual presentations such as dysphagia, foot-drop, "dropped-head," or camptocormia due to weakness of the paraspinal muscles, while scapuloperoneal or facioscapulohumeral patterns of weakness have also been reported. ${ }^{3}$ Sporadic IBM with the atypical presentation was underdiagnosed or misdiagnosed, which will affect the quality of life. In such a case, muscle biopsy plays an important role in the diagnosis.

published online

November 22, 2021
DOI https://doi.org/ $10.1055 / \mathrm{s}-0041-1739230$. ISSN 2582-4287.

\section{Case Report}

A 55-year-old male presented with complaints of insidious onset of symmetrical flaccid weakness (proximal more than distal) involving bilateral upper and lower limb for the past 6 months. On further questioning, the patient described difficulty in standing and getting up from squatting or sitting posture. He also developed difficulty in climbing stairs, raising hands above the head, and difficulty in combing hairs. He also had erythematous skin rash over bilateral nasolabial fold, anterior aspect of chest, and back of the neck for the past 6 months.

On examination, he had the following pattern on muscular strength testing: neck flexors were $5 / 5$, bilateral shoulder abduction (supraspinatus) was $3 / 5$; bilateral shoulder adduction (pectoralis major and minor) was $3 / 5$; bilateral shoulder flexion and extension (deltoid) were $3 / 5$, bilateral elbow flexion (biceps) and extension(triceps) were 3/5; bilateral wrist flexion(flexor carpi ulnaris, flexor carpi radialis, flexor digitorum) and wrist extension (extensor carpi
(C) 2021. Nitte (Deemed to be University). All rights reserved. This is an open access article published by Thieme under the terms of the Creative Commons Attribution-NonDerivative-NonCommercial-License, permitting copying and reproduction so long as the original work is given appropriate credit. Contents may not be used for commercial purposes, or adapted, remixed, transformed or built upon. (https://creativecommons.org/ licenses/by-nc-nd/4.0/)

Thieme Medical and Scientific Publishers Pvt. Ltd., A-12, 2nd Floor, Sector 2, Noida-201301 UP, India 


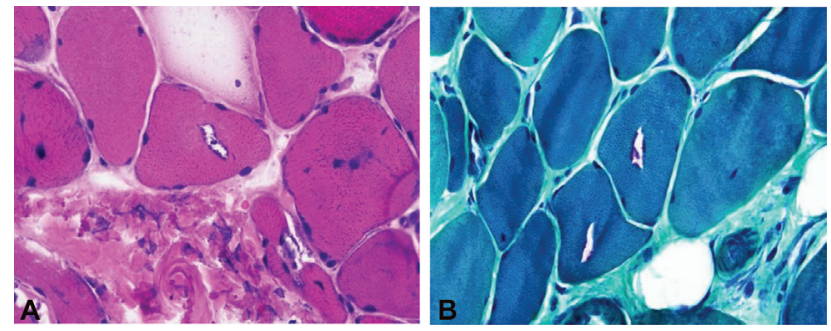

Fig. 1 (A and B) Biopsy showing atrophy, multifocal rimmed vacuoles, endomysial lymphonuclear infiltrates involving the nonnecrotizing fibers.

ulnaris, extensor carpi radialis, extensor indices) were $-4 / 5$; and hand grip (finger flexors) strength was around 60 to $70 \%$ on both sides. In his lower extremities, bilateral hip flexion (iliopsoas), hip extension (gluteus maximus), hip abduction (gluteus medius and minimus), and hip adductors strength were $3 / 5$; bilateral knee flexion(hamstrings) and knee extension(quadriceps) were $3 / 5$; bilateral ankle dorsiflexion (tibialis anterior) and ankle plantarflexion (gastrocsoleus) were- $4 / 5$. His reflexes were intact throughout, and he had no sensory disturbances.

Laboratory studies revealed an elevated total creatine kinase level of 1,164 IU/L (normal 25-200 IU/L) and other blood parameters are within normal limits. Electromyography showed a myopathic pattern of changes. Nerve conduction study showed motor-sensory axonal demyelinating changes noted in the upper limb and lower limb.

Based on the following investigations and clinical manifestations, the patient was initially diagnosed as dermatomyositis/polymyositis. Myositis antigen extended profile was done. Mi- $2 \beta$ and Jo- 1 came as borderline (i.e., increased but negative) specific for dermatomyositis and polymyositis. A skin biopsy was taken to diagnose dermatomyositis, but it revealed as nonspecific dermatitis. Therefore, we sought to investigate the other causes of inflammatory myopathies. Hence, we done a muscle biopsy from vastus lateralis (-Fig. 1A, B), which showed atrophy, multifocal rimmed vacuoles, endomysial lymphonuclear infiltrates involving the nonnecrotizing fibers suggestive of Sporadic IBM.

Magnetic resonance imaging of right shoulder and proximal thigh showed diffuse T2-weighted short tau inversion recovery (-Fig. 2A-D) hyperintensity and intramuscular T1 high signal intensities in these muscles suggestive of chronic inflammation with fat infiltration. In addition, a nerve biopsy from the sural nerve showed axonal neuropathy.

Finally, the patient was diagnosed with sporadic IBM with an atypical presentation, and the patient was started on corticosteroids. He improved symptomatically and was on regular follow-up.

\section{Discussion}

Sporadic (IBM) is one of several chronic adult inflammatory myopathies. Its prevalence varies, but it may be as high as 35 per 1 million adults over age 50 , with a slight male predominance. ${ }^{3}$ IBM typically manifests as slowly progressive weakness of quadriceps muscle more than hip flexors leading to frequent falls or difficulty in standing and next common problem would be finger flexor weakness leading to loss of dexterity. ${ }^{4}$

However, all sporadic IBM may not have the classic presentation of distal arm and proximal leg involvement. In this case, it can present with symmetrical weakness where the proximal group of muscles involved more than the distal group of muscles without complications like dysphagia and most commonly misdiagnosed as polymyositis or dermatomyositis.

In such case, muscle biopsy and imaging of muscle play an important role in diagnosis. The recent identification of a serum autoantibody against anti-5'-nucleotidase, cytosolic IA in patients with sporadic IBM has offered a new clinical tool.

Anticytosolic 5'-nucleotidase is a highly specific diagnostic marker for IBM among patients with myopathy. Other blood biomarkers for IBM include an abnormal population of large granular lymphocytes on flow cytometry and a reduced CD4/CD8 ratio with an increased CD8 count.

High-dose corticosteroids are considered the first-line treatment. Unfortunately, IBM does not typically respond to any known immunotherapies. The mainstay of treatment is physical and occupational therapy to improve function and swallowing therapy. Patients with dysphagia may benefit from intravenous immunoglobulin therapy, along with esophageal balloon dilation or cricopharyngeal myotomy. Most patients require a wheelchair within 10 to 15 years of onset of symptoms. Life expectancy is not significantly altered in IBM. ${ }^{5}$
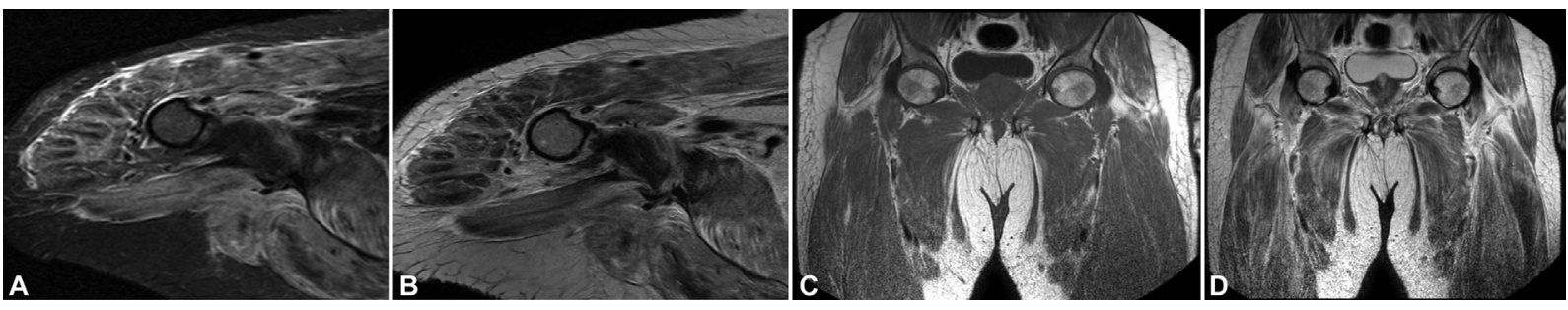

Fig. 2 (A) T2-weighted short tau inversion recovery imaging shows diffuse hyperintensity noted in shoulder girdle muscles and edema noted in the intramuscular plane. (B) T2-weighted axial image shows high intensity in the muscle suggestive of chronic inflammation with fat replacement/ infiltration. (C) T1-weighted image of thigh shows high intensities in the intramuscular plane. (D) T2-weighted image of thigh shows bilateral symmetrical high signal intensity noted in all visualized muscles. 


\section{Conclusion}

Sporadic IBM with atypical presentation is still difficult to diagnose and unfortunately remains frequently misdiagnosed; in such cases, muscle biopsy and muscle imaging play an important role in diagnosis. Although sporadic IBM is rare and without effective therapy, accurate diagnosis is crucial to providing adequate patient counseling and information about the prognosis and course of the disease. Patients with IBM are highly motivated and should be encouraged to participate in clinical trials.

\section{Conflict of Interest}

None declared.

\section{References}

1 Shroff S, Pleitez M. Atypical presentation of sporadic inclusion body myositis.(P5. 062). Neurology 2015;84(14 Supplement): P5.062

2 Weihl CC. Sporadic inclusion body myositis and other rimmed vacuolar myopathies. Continuum (Minneap Minn) 2019;25(06): 1586-1598

3 Mastaglia FL. Sporadic inclusion body myositis: variability in prevalence and phenotype and influence of the MHC. Acta Myol 2009;28(02):66-71

4 Dimachkie MM, Barohn RJ. Inclusion body myositis. Curr Neurol Neurosci Rep 2013;13(01):321

5 Jameson JL. Harrison's Principles of Internal Medicine. Philadelphia, PA: McGraw-Hill Education; 2018 